\title{
Will GRPR Compete with PSMA as a Target in Prostate Cancer?
}

\author{
Andrei Iagaru \\ Division of Nuclear Medicine and Molecular Imaging, Department of Radiology, Stanford University, Stanford, California
}

I maging of prostate cancer (PCa) plays an important role in guiding biopsy at initial diagnosis, in accurately evaluating the extent of disease, in assessing sites of recurrent disease, and in monitoring response to treatment. A variety of PET radiopharmaceuticals for $\mathrm{PCa}$ are in clinical trials or available commercially.

Prostate-specific membrane antigen (PSMA) is a transmembrane protein that is highly overexpressed on almost all PCas (1). Only 5\%$10 \%$ of primary PCa lesions have been shown to be PSMA-negative (2), making this class of radiopharmaceutical suitable for diagnosis of primary PCa and for initial staging $(3,4)$. Use for noninvasive tumor grading has also been reported (3). High accuracy has been reported for use in detecting biochemical recurrence, even at very low prostate-specific antigen levels $(5,6)$. However, these promising agents do not detect all recurrences $(5,7)$, and other types of cancer also express PSMA $(8,9)$. False-positive findings have also been reported using PSMA agents (10-12). Given the widespread adoption of PSMA radiopharmaceuticals and their excellent performance at all stages of PCa, is there a need or a role for another class of PET radiopharmaceutical targeting other biologic processes in $\mathrm{PCa}$ ?

Gastrin-releasing peptide receptor (GRPR) is a G-protein-coupled receptor overexpressed in many types of cancer, such as PCa, breast cancer, and small cell lung cancer (13). It belongs to the bombesin receptor family. Bombesin is a 14-mer peptide (Pyr-Gln-Arg-LeuGly-Asn-Gln-Trp-Ala-Val-Gly-His-Leu-Met- $\mathrm{NH}_{2}$ ) that binds with high affinity to the GRPR (14). Clinically translated GRPR antagonist PET radiopharmaceuticals include ${ }^{68} \mathrm{Ga}-\mathrm{RM} 2,{ }^{68} \mathrm{Ga}-$ SB3, ${ }^{68} \mathrm{Ga}-\mathrm{NeoBOMB} 1,{ }^{18} \mathrm{~F}-\mathrm{BAY}-864367$, and ${ }^{64} \mathrm{Cu}-\mathrm{CB}-\mathrm{TE} 2 \mathrm{~A}-$ AR06. They have a stable biodistribution in healthy volunteers (15) and mean effective doses comparable to those of other radiopharmaceuticals $(16,17)$. Data are encouraging regarding their potential for the initial diagnosis of $\mathrm{PCa}(15-19)$, for the assessment of biochemical recurrence $(15,16,18-23)$, and for the theranostic approach (24). The lack of salivary or lacrimal gland uptake and the low hepatobiliary clearance give them an advantage. The high pancreatic uptake may be of concern to some; however, although initial uptake is high, it decreases rapidly whereas tumor radioactivity is retained longer (24). A comparison of ${ }^{68} \mathrm{Ga}$-RM2 PET scans before and after treatment is shown in Figure 1, and comparisons of paired ${ }^{68} \mathrm{Ga}-\mathrm{PSMA}-11$ and ${ }^{68} \mathrm{Ga}-\mathrm{RM} 2$ scans are shown in Figure 2.

Received Aug. 28, 2017; revision accepted Sep. 13, 2017.

For correspondence or reprints contact: Andrei lagaru, Division of Nuclear Medicine and Molecular Imaging, Department of Radiology, Stanford University, 300 Pasteur Dr., H2200, Stanford, CA 94305.

E-mail: aiagaru@stanford.edu

Published online Sep. 28, 2017.

COPYRIGHT (c) 2017 by the Society of Nuclear Medicine and Molecular Imaging. DOI: 10.2967/jnumed.117.198192
Although the overexpression of PSMA is ubiquitous in PCa, it is not universal and there will be lesions not detected by PSMAtargeted imaging in different risk classes or stages of disease. The influence of GRPR expression on cancer grade and stage is not clear. Nagasaki et al. (25) found that GRPR expression was positively correlated with Gleason score, but another study found that GRPR expression was inversely correlated with Gleason score, preoperative prostate-specific antigen concentration, and tumor size (26). Therefore, the indication (i.e., low- vs. intermediate- vs high-risk, and early vs. late stage of disease) for the use of PSMAvs. GRPR-targeted imaging or both is an active focus of research.

Another unsettled question is whether the uptake is specific for (prostate) cancer. High uptake was reported in primary $\mathrm{PCa}$ lesions and metastases $(16,18,23)$, but benign prostate hypertrophy resulted in false-positive findings in some cases (16). This may be avoided by the development of appropriate metrics of image interpretation. In our experience with ${ }^{68} \mathrm{Ga}-\mathrm{RM} 2$, all 7 biopsied lesions (prostate, lymph nodes, liver, and lung nodule) were confirmed PCa (22). As GRPR-targeting radiopharmaceuticals are evaluated in more patients, it is likely that more reports of non-cancer-specific uptake will emerge, as with any new class of radiopharmaceutical.

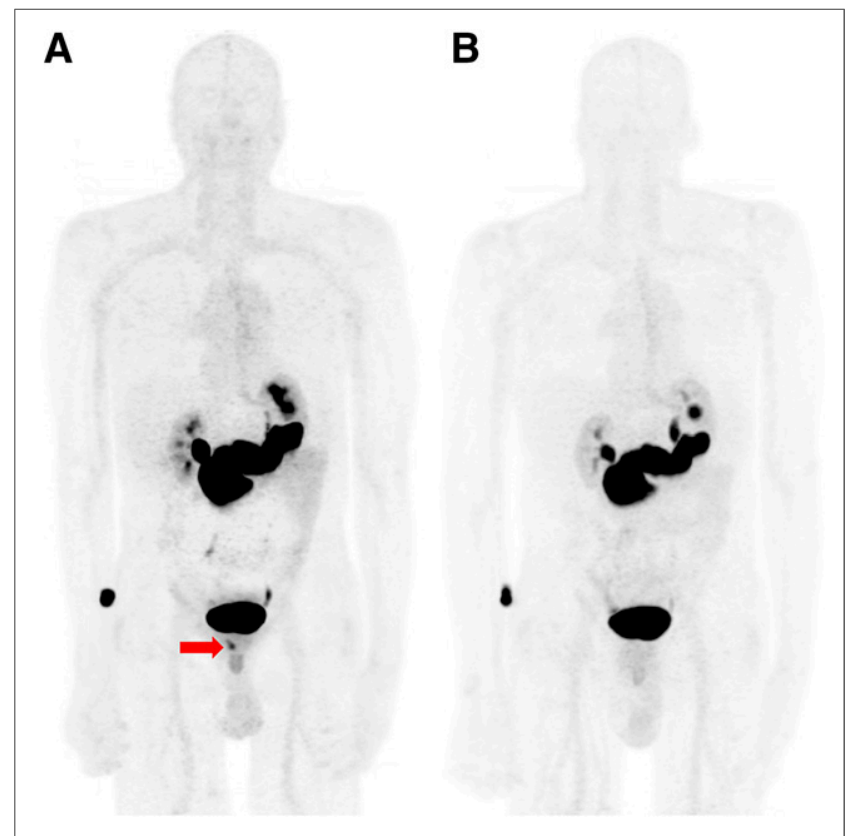

FIGURE 1. 72-y-old man with history of Gleason $3+4$ PCa treated with radiation and hormonal therapy, presenting with prostate-specific antigen level of $3.7 \mathrm{ng} / \mathrm{mL}$. Maximum-intensity-projection ${ }^{68} \mathrm{Ga}-\mathrm{RM} 2$ PET images before (A) and after $(B)$ radiation treatment demonstrate resolution of prostate bed uptake (arrow). 


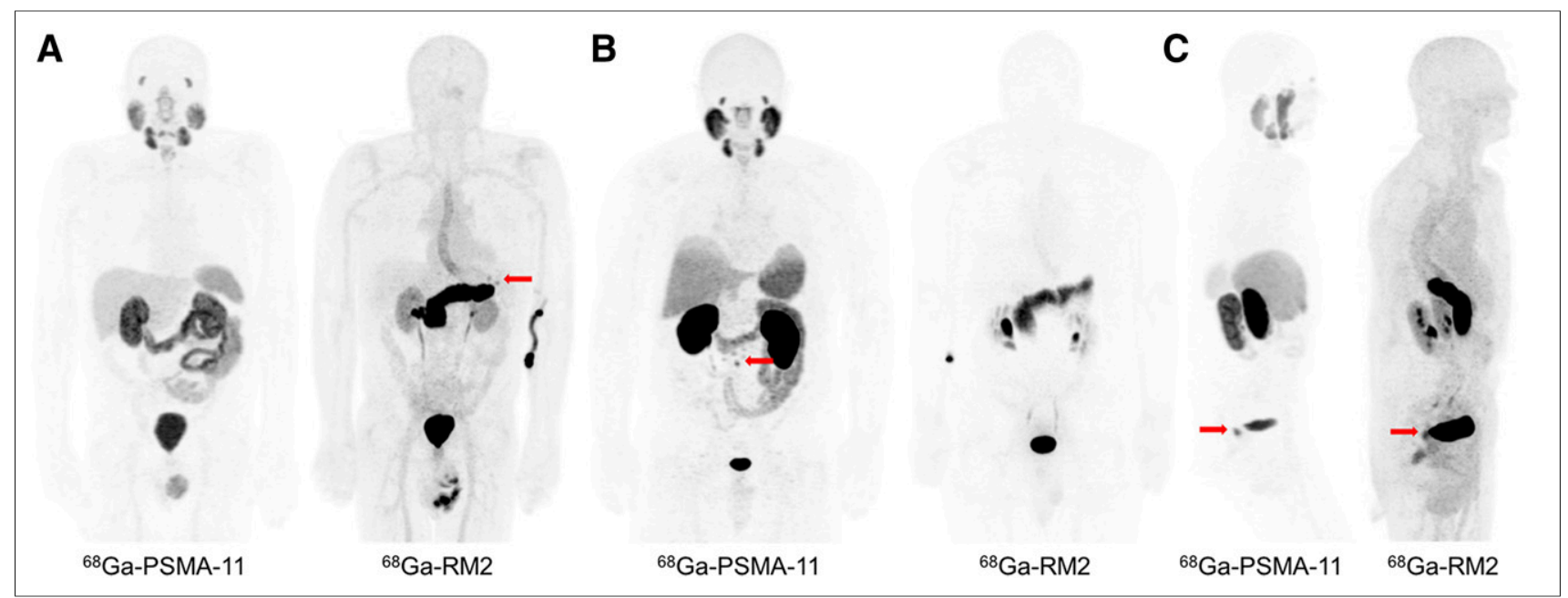

FIGURE 2. Maximum-intensity-projection PET images from ${ }^{68} \mathrm{Ga}-\mathrm{PSMA}-11$ and ${ }^{68} \mathrm{Ga}-\mathrm{RM} 2 \mathrm{PET}$ scan pairs show no uptake on ${ }^{68} \mathrm{Ga}-\mathrm{PSMA}-11 \mathrm{PET}$ and focal uptake (arrow) in retroperitoneal lymph nodes on ${ }^{68} \mathrm{Ga}-\mathrm{RM} 2 \mathrm{PET}$ (A), focal uptake (arrow) in retroperitoneal lymph nodes on ${ }^{68} \mathrm{Ga}-\mathrm{PSMA}-11$ PET and no uptake on ${ }^{68} \mathrm{Ga}-\mathrm{RM} 2 \mathrm{PET}(\mathrm{B})$, and focal uptake (arrows) in seminal vesicle on both ${ }^{68} \mathrm{Ga}-\mathrm{PSMA}-11 \mathrm{PET}$ and ${ }^{68} \mathrm{Ga}-\mathrm{RM} 2 \mathrm{PET}(\mathrm{C})$.

There are limitations to the data supporting the use of GRPRtargeting PET peptides, including small clinical studies, limited gold standards, and limited outcome data based on their use. Larger studies are needed to confirm preliminary results. However, given the complexity of the biologic processes identified in $\mathrm{PCa}$, it is unlikely that any one group of imaging agents will provide all the answers needed to offer the best care for these patients. Therefore, the continued development and introduction to clinical use of GRPR-targeting PET radiopharmaceuticals will continue, if not as direct competitors of PSMA-targeting tracers, then as complementary diagnostic and therapeutic approaches. The sum is better than the parts after all.

\section{DISCLOSURE}

No potential conflict of interest relevant to this article was reported.

\section{REFERENCES}

1. Silver DA, Pellicer I, Fair WR, Heston WD, Cordon-Cardo C. Prostate-specific membrane antigen expression in normal and malignant human tissues. Clin Cancer Res. 1997;3:81-85.

2. Budäus L, Leyh-Bannurah SR, Salomon G, et al. Initial experience of ${ }^{68} \mathrm{Ga}-$ PSMA PET/CT imaging in high-risk prostate cancer patients prior to radical prostatectomy. Eur Urol. 2016;69:393-396.

3. Koerber SA, Utzinger MT, Kratochwil C, et al. ${ }^{68} \mathrm{Ga}$-PSMA11-PET/CT in newly diagnosed carcinoma of the prostate: correlation of intraprostatic PSMA uptake with several clinical parameters. J Nucl Med. June 15, 2017 [Epub ahead of print].

4. Fendler WP, Schmidt DF, Wenter V, et al. ${ }^{68} \mathrm{Ga}$-PSMA PET/CT detects the location and extent of primary prostate cancer. J Nucl Med. 2016;57:1720-1725.

5. Eiber M, Maurer T, Souvatzoglou M, et al. Evaluation of hybrid ${ }^{68} \mathrm{Ga}-\mathrm{PSMA}$ ligand PET/CT in 248 patients with biochemical recurrence after radical prostatectomy. J Nucl Med. 2015;56:668-674.

6. Afshar-Oromieh A, Avtzi E, Giesel FL, et al. The diagnostic value of PET/CT imaging with the ${ }^{68} \mathrm{Ga}$-labelled PSMA ligand HBED-CC in the diagnosis of recurrent prostate cancer. Eur J Nucl Med Mol Imaging. 2015;42:197-209.

7. Rowe SP, Gage KL, Faraj SF, et al. ${ }^{18} \mathrm{~F}$-DCFBC PET/CT for PSMA-based detection and characterization of primary prostate cancer. J Nucl Med. 2015;56:1003-1010.

8. Sathekge M, Lengana T, Modiselle M, et al. ${ }^{68}$ Ga-PSMA-HBED-CC PET imaging in breast carcinoma patients. Eur J Nucl Med Mol Imaging. 2017;44:689-694.

9. Rhee H, Blazak J, Tham CM, et al. Pilot study: use of gallium-68 PSMA PET for detection of metastatic lesions in patients with renal tumour. EJNMMI Res. 2016;6:76.

10. Hermann RM, Djannatian M, Czech N, Nitsche M. Prostate-specific membrane antigen PET/CT: false-positive results due to sarcoidosis? Case Rep Oncol. 2016;9:457-463.
11. Sasikumar A, Joy A, Nanabala R, Pillai MR, T AH. ${ }^{68}$ Ga-PSMA PET/CT falsepositive tracer uptake in Paget disease. Clin Nucl Med. 2016;41:e454-e455.

12. Noto B, Vrachimis A, Schafers M, Stegger L, Rahbar K. Subacute stroke mimicking cerebral metastasis in ${ }^{68} \mathrm{Ga}-\mathrm{PSMA}-\mathrm{HBED}-\mathrm{CC}$ PET/CT. Clin Nucl Med. 2016;41:e449-e451.

13. Mansi R, Fleischmann A, Macke HR, Reubi JC. Targeting GRPR in urological cancers: from basic research to clinical application. Nat Rev Urol. 2013;10:235-244.

14. Jensen RT, Battey JF, Spindel ER, Benya RV. International Union of Pharmacology. LXVIII. Mammalian bombesin receptors: nomenclature, distribution, pharmacology, signaling, and functions in normal and disease states. Pharmacol Rev. 2008;60:1-42.

15. Roivainen A, Kahkonen E, Luoto P, et al. Plasma pharmacokinetics, whole-body distribution, metabolism, and radiation dosimetry of ${ }^{68} \mathrm{Ga}$ bombesin antagonist BAY 86-7548 in healthy men. J Nucl Med. 2013;54:867-872.

16. Kähkönen E, Jambor I, Kemppainen J, et al. In vivo imaging of prostate cancer using $\left[{ }^{68} \mathrm{Ga}\right]-$ labeled bombesin analog BAY86-7548. Clin Cancer Res. 2013;19:54345443.

17. Wieser G, Mansi R, Grosu AL, et al. Positron emission tomography (PET) imaging of prostate cancer with a gastrin releasing peptide receptor antagonist: from mice to men. Theranostics. 2014;4:412-419.

18. Nock BA, Kaloudi A, Lymperis E, et al. Theranostic perspectives in prostate cancer with the gastrin-releasing peptide receptor antagonist NeoBOMB1: preclinical and first clinical results. $J$ Nucl Med. 2017;58:75-80.

19. Sah BR, Burger IA, Schibli R, et al. Dosimetry and first clinical evaluation of the new ${ }^{18}$ F-radiolabeled bombesin analogue BAY 864367 in patients with prostate cancer. J Nucl Med. 2015;56:372-378.

20. Minamimoto R, Hancock S, Schneider B, et al. Pilot comparison of ${ }^{68}$ Ga-RM2 PET and ${ }^{68} \mathrm{Ga}$-PSMA-11 PET in patients with biochemically recurrent prostate cancer. J Nucl Med. 2016;57:557-562.

21. Wieser G, Popp I, Christian Rischke H, et al. Diagnosis of recurrent prostate cancer with PET/CT imaging using the gastrin-releasing peptide receptor antagonist ${ }^{68} \mathrm{Ga}-$ RM2: preliminary results in patients with negative or inconclusive $\left[{ }^{18} \mathrm{~F}\right]$ fluoroethylcholine-PET/CT. Eur J Nucl Med Mol Imaging. 2017;44:1463-1472.

22. Harrison C, Sonni I, Loening A, Vasanawala S, Iagaru A. Detection of recurrent prostate cancer using ${ }^{68} \mathrm{Ga}-\mathrm{RM} 2 \mathrm{PET} / \mathrm{MRI}$ in patients with negative conventional imaging [abstract]. J Nucl Med. 2017;58(suppl 1):711.

23. Maina T, Bergsma H, Kulkarni HR, et al. Preclinical and first clinical experience with the gastrin-releasing peptide receptor-antagonist $\left[{ }^{68} \mathrm{Ga}\right] \mathrm{SB} 3$ and PET/CT. Eur J Nucl Med Mol Imaging. 2016;43:964-973.

24. Dalm SU, Bakker IL, de Blois E, et al. ${ }^{68} \mathrm{Ga} /{ }^{177} \mathrm{Lu}-\mathrm{NeoBOMB} 1$, a novel radiolabeled GRPR antagonist for theranostic use in oncology. J Nucl Med. 2017;58:293-299.

25. Nagasaki S, Nakamura Y, Maekawa T, et al. Immunohistochemical analysis of gastrin-releasing peptide receptor (GRPR) and possible regulation by estrogen receptor $\beta \mathrm{cx}$ in human prostate carcinoma. Neoplasma. 2012;59:224-232.

26. Beer M, Montani M, Gerhardt J, et al. Profiling gastrin-releasing peptide receptor in prostate tissues: clinical implications and molecular correlates. Prostate. 2012;72:318-325. 